\title{
Transatlantica
}

Revue d'études américaines. American Studies Journal

\section{Enfin ! At long last !}

\section{(2) OpenEdition}

1 Journals

Édition électronique

URL : http://journals.openedition.org/transatlantica/630

DOI : 10.4000/transatlantica.630

ISSN : 1765-2766

Éditeur

AFEA

Référence électronique

«Enfin! At long last! », Transatlantica [En ligne], 1 | 2003, mis en ligne le 30 mars 2006, consulté le 29 avril 2021. URL : http://journals.openedition.org/transatlantica/630 ; DOI : https://doi.org/10.4000/ transatlantica.630

Ce document a été généré automatiquement le 29 avril 2021.

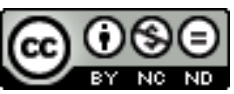

Transatlantica - Revue d'études américaines est mis à disposition selon les termes de la licence Creative Commons Attribution - Pas d'Utilisation Commerciale - Pas de Modification 4.0 International. 


\section{Enfin! At long last!}

1 Ce qui devait être le cadeau de Noël de l'AFEA est devenu un oeuf de Pâques... Aussi, je souhaiterais avant toute chose demander à nos lecteurs, et aux auteurs qui nous ont fait confiance, de bien vouloir excuser ce retard de publication. Les causes en sont nombreuses ; j'en retiendrai trois.

- Nous voulions assurer une qualité des textes publiés. Le tri a donc été sévère, les ré-écritures aussi et je tiens à remercier chaleureusement tous ceux qui, dans le comité et à l'extérieur, anciens ou nouveaux membres, nous ont aidés à relire les contributions, ont suggéré de nombreuses améliorations de fond ou de forme ; et bien sûr les auteurs qui nous ont fait confiance. J'en profite pour dire à ceux qui nous ont soumis des articles auxquels nous n'avons pas encore pu donner de suite positive que nous espérons bien les publier dans les prochaines livraisons. TransatlanticA est un atelier d'études américaines dont la vocation est de s'élargir.

- Nous souhaitions maintenir la notion de livraison, comme pour une revue papier classique (à vous de nous dire si c'est une formule qui s'applique bien aux nouveaux modes de lecture produits par le web). Ce choix s'oppose à celui d'une publication d'articles au fur et à mesure de leur achèvement qui risquerait de transformer la revue en simple archive de textes où des lecteurs viendraient butiner. Ce n'est pas notre conception car pour nous, une revue est avant tout un projet scientifique.

- Enfin, nous voulions privilégier les dossiers, parfois issus de journées d'études, ce qui impose des contraintes fortes dans la composition du numéro. TransatlanticA constitue pour les recherches en cours à la fois un niveau d'élaboration supplémentaire et offre une certaine informalité qui garde à la publication sa nature d'échange. L'appel est donc lancé.

Parlez-nous de vos projets scientifiques, proposez-nous des contributions imaginatives, et menons ensemble ce dialogue américaniste que nous appelons de nos voeux.

2 Mais, pour conduire pleinement ce dialogue avec nos amis et collègues européens, américains et autres, il est clair que se pose le problème de la langue d'échange. Le dossier de ce numéro, sur le syndicalisme américain, est presque exclusivement en anglais. Nous serons amenés à nous poser encore cette question cruciale.

3 C'est aussi pour innover tout en conservant des repères connus, que nous avons développé la rubrique «Varia » avec une responsable de rubrique, Géraldine Chouard, 
qui a fait un véritable travail de collecte et d'élaboration pour cet espace dédié aux formes critiques brèves.

4

Enfin, nous continuons le travail de publication de travaux de doctorants amorcé dans les 2 premiers numéros. C'est une des vocations de TransatlanticA dans sa dimension d'ouvroir à laquelle l'équipe de rédaction tient beaucoup. La revue est une oeuvre éphémère qui constitue un modeste mais indispensable jalon dans un long débat, un outil dans la construction permanente d'une compréhension critique des Etats-Unis. N'hésitez donc pas à reprendre la balle au bond et à nous adresser un texte, un développement, une réponse. Inventons les nouvelles formes du travail scientifique ! 